\title{
Excessive periostin expression and Th2 response in patients with nasal polyps: association with asthma
}

\author{
Yi Wei ${ }^{1,2 \#}$, Renqiang $\mathrm{Ma}^{1,2 \#}$, Jia Zhang ${ }^{1,3}$, Xingmei $\mathrm{Wu}^{1,2}$, Guodong $\mathrm{Yu}^{4}$, Xianting $\mathrm{Hu}^{3}$, Jian $\mathrm{Li}^{1,2}$, \\ Zhuofu Liu ${ }^{3}$, Wendong $\mathrm{Ji}^{1}$, Huabin $\mathrm{Li}^{3}$, Weiping Wen ${ }^{1,2}$
}

${ }^{1}$ Otorhinolaryngology Hospital, The First Affiliated Hospital of Sun Yat-sen University, Guangzhou 510080, China; ${ }^{2}$ Guangzhou Key Laboratory of Otorhinolaryngology, Guangzhou 510000, China; ${ }^{3}$ Department of Otolaryngology, Head and Neck Surgery, Affiliated Eye, Ear, Nose and Throat Hospital, Fudan University, Shanghai 200031, China; ${ }^{4}$ Department of Otolaryngology, Affiliated Hospital of Guizhou Medical University, Guiyang 550000, China

Contributions: (I) Conception and design: Y Wei, H Li, W Wen; (II) Administrative support: R Ma, W Ji; (III) Provision of study materials or patients: Y Wei, X Wu, H Li; (IV) Collection and assembly of data: Y Wei, J Zhang, G Yu, X Hu; (V) Data analysis and interpretation: Y Wei, J Li, Z Liu; (VI) Manuscript writing: All authors; (VII) Final approval of manuscript: All authors.

\#These authors contributed equally to this work.

Correspondence to: Weiping Wen. The First Affiliated Hospital of Sun Yat-sen University, No. 58, Zhongshan $2^{\text {nd }}$ Road, Guangzhou 510080 , China. Email: wenwp@mail.sysu.edu.cn; Weidong Ji. The center for Translational Medicine, The First Affiliated Hospital of Sun Yat-sen University, No. 58, Zhongshan 2nd Road, Guangzhou 510080, China. Email: wdji2008@126.com; Huabin Li. Department of Otolaryngology, Head and Neck Surgery, Affiliated Eye, Ear, Nose and Throat Hospital, Fudan University, No. 83, Fenyang Road, Shanghai 200031, China. Email: allergyli@163.com.

Background: Periostin has been shown to be upregulated in chronic rhinosinusitis with nasal polyps (CRSwNP), especially in the CRSwNP patients with asthma. However, the underlying mechanism that how periostin contributes to the polyp genesis remains unclear.

Methods: In this study, we collected 63 CRSwNP patients' nasal polyps (NPs) and 25 control subjects' uncinated tissues. The expressions of periostin, thymic stromal lymphopoietin (TSLP), and other proinflammatory cytokines were examined using IHC staining, qRT-PCR, Western blot (WB), ELISA and FACS. The eosinophil infiltration, phenotype profiles and clinical characteristics of 2 NP subtypes (eosinophilic and non-eosinophilic) were evaluated. We examined the effects and mechanisms of periostin on human nasal epithelial cells cultured at air-liquid interface (ALI).

Results: The expressions of periostin in NPs with asthma were higher than without asthma and the control nasal mucosa and positively associated with the TSLP $(\mathrm{P}<0.05)$. And the periostin levels was positively associated with the basement membrane thickness, goblet cell hyperplasia and tissue eosinophilia polyp tissues, as well as the clinical parameters (computed tomography scores, polyp size, and polyp recurrence after endoscopic surgery). In vitro experiments show that type 2 T-helper (Th2) cytokines interleukin-4 (IL-4), IL-13 and TGF- $\beta 1$ stimulates epithelial cells derived from polyp tissues to produce periostin through ERK and STAT6 signal pathways $(\mathrm{P}<0.05)$. Autocrine or recombinant periostin activates epithelial cells to produce TSLP via NF- $\kappa$ B signal pathways $(\mathrm{P}<0.05)$. The supernatant of periostin-treated epithelial cells activates dendritic cells (DCs), which subsequently induce naïve $\mathrm{T}$ cells to differentiate into Th2 cells and express IL-4 and IL-13.

Conclusions: Our findings indicate periostin may play an important role in the polyp genesis, which can be considered as a therapeutic target for the management of CRSwNP.

Keywords: Chronic rhinosinusitis with nasal polyps (CRSwNP); asthma; periostin; thymic stromal lymphopoietin (TSLP); eosinophil infiltration; type 2 T-helper cell response

Submitted May 28, 2018. Accepted for publication Oct 31, 2018.

doi: $10.21037 /$ jtd.2018.11.12

View this article at: http://dx.doi.org/10.21037/jtd.2018.11.12 


\section{Introduction}

Chronic rhinosinusitis with nasal polyps (CRSwNP) is the nasal mucosa and paranasal sinuses inflammation at least 12 weeks duration, with visible nasal polyps (NPs) (1). NPs has been characterized in histologic studies by enhanced type 2 T-helper (Th2) response and eosinophilic inflammation (2-5). Although airway epithelial cells secrete Th2 pro-inflammatory cytokines such as thymic stromal lymphopoietin (TSLP), IL-25, and IL-33 playing a pivotal role in the pathogenesis of CRSwNP $(6,7)$, the signaling mechanisms responsible for cytokines production of epithelial cells of NPs and relevant effects between epithelial cells activation and immune cells are not fully understood.

Periostin is a matricellular protein belonging fasciclin family, which are involved not only in tissue developing and remodeling but also in the Th2-biased inflammation and eosinophilic infiltration of airway and skin allergic diseases, such as asthma $(8,9)$. Recent studies have shown, periostin in NPs is mainly produced by epithelial cells and regulated by several mediators including Th2 cytokines IL-4, IL$13(10,11)$. Periostin is able to stimulate epithelial cells to secret TSLP, which may important in activating Th2 responses $(12,13)$. However, the underlying mechanism that how periostin contributes to the polyp genesis remains unclear. To address this issue, we investigated the expression of periostin in polyp tissues, and evaluated its association with asthma and the clinical severity of CRSwNP patients, and examined the possible molecular mechanisms.

\section{Methods}

\section{Patients and tissue samples}

Sixty-three CRSwNP patients and 25 control subjects were randomly recruited from the First Affiliated Hospital of Sun Yat-sen University in Guangzhou. The diagnosis of CRSwNP and computed tomography (CT) scan of the paranasal cavities were made according to the international consensus (EPOS 2012) (1). The atopic status of all patients was evaluated by skin prick tests. The pneumologist diagnosed asthma by airway responsiveness and disease history. The $14 \mathrm{CRSwNP}$ patients with asthma contain 2 patients are intermittent $\left(\% \mathrm{FEV}_{1}\right.$ of predicted $\geq 80 \%, \mathrm{FEV}_{1}$ variability $<20 \%), 4$ patients are Mild persistent $\left(\% \mathrm{FEV}_{1}\right.$ of predicted $\geq 80 \%, \mathrm{FEV}_{1}$ variability $20-30 \%$ ), 4 patients are Moderate persistent (\% $\mathrm{FEV}_{1}$ of predicted $60-80 \%, \mathrm{FEV}_{1}$ variability $>30 \%), 4$ patients are Severe persistent $\left(\% \mathrm{FEV}_{1}\right.$
Table 1 Demographics of subjects

\begin{tabular}{|c|c|c|}
\hline Demographics & Control subjects & $\begin{array}{c}\text { Patients with } \\
\text { CRSwNP }\end{array}$ \\
\hline No. & 25 & 63 \\
\hline Tissue sampled & UT & NP \\
\hline Age, mean (SD) (years) & $34.79(10.68)$ & $35.03(10.97)$ \\
\hline Atopy, no. (\%) & $4(16.00)$ & $13(20.63)^{\star}$ \\
\hline Asthma, no. (\%) & 0 & $14(22.22)$ \\
\hline Aspirin sensitivity, no. (\%) & 0 & $4(6.35)$ \\
\hline Sex (male/female) & $14 / 11$ & $33 / 30$ \\
\hline CT scores, mean (SD) & NA & $15.79(10.03)$ \\
\hline Total IgE, mean (SD) (IU/mL) & $12.52(4.79)$ & $91.58(50.67)^{\star}$ \\
\hline $\begin{array}{l}\text { Blood eosinophils, mean (SD) } \\
\left(10^{9} / \mathrm{L}\right)\end{array}$ & $0.02(0.05)$ & $0.35(0.39)^{*}$ \\
\hline \multicolumn{3}{|l|}{ Methodologies used } \\
\hline Tissue ELISA & 25 & 63 \\
\hline Tissue IHC & 25 & 34 \\
\hline Tissue mRNA & 25 & 63 \\
\hline Tissue WB & 7 & 9 \\
\hline
\end{tabular}

of predicted $<60 \%, \mathrm{FEV}_{1}$ variability $\left.>30 \%\right)$. Nine patients are eosinophilic asthma phenotype (blood eosinophil counts of greater than $300 / \mu \mathrm{L}$, sputum eosinophil counts of greater than 2\%), 5 patients are non-eosinophilic asthma. During endoscopic surgery, the following nasal tissues were sampled: polyp tissues from patients with CRSwNP and the uncinated process tissues of control patients with septoplasty due to anatomical variations. The demographic data of all subjects enrolled in this study are listed in Table 1.

NP size was divided into 1, 2 and 3 grades: (I) polyp size was limited in the middle meatus; (II) polyps size was the range from the middle meatus to the inferior turbinate; (III) polyps size congested the inferior meatus. Newborn NPs after surgical resection 12 weeks, were regarded as the recurrence. CRSwNP patients divided into eosinophilic and non-eosinophilic subsets according to eosinophilic cells counted in hematoxylin and eosin (HE) staining. The cutoff value for the eosinophilic phenotype was set at 7 eosinophilic cells/HPF (400x), which is equivalent to 
the mean value in the healthy control subjects (2.21) plus 2 times the standard deviation (SD) $(2.37 \times 2=4.74$; cutoff value, $2.21+4.74=6.95)$.

Nasal tissues were used for RNA, protein extraction, the histologic assessment and isolated human nasal epithelial cells (HNECs). HNECs were cultured to assess the effect of inflammatory cytokines on periostin and TSLP expression in vitro.

\section{Histologic staining}

$\mathrm{HE}$, Alcian new blue and periodic acid-Schiff (AB-PAS), and Masson trichrome staining were performed according to the manufacturer's instructions. Immunohistochemically staining for periostin, TSLP was performed as previously described (14,15). Rabbit anti-periostin antibody, Abcam, ab14041, 1:500; Rabbit anti-periostin antibody, Abcam, ab79946, $0.1 \mu \mathrm{g} / \mathrm{mL}$; rabbit anti-TSLP antibody, 1:100, Abcam, ab47943 were using as the primary antibodies according to the manufacturer's instructions. Each section was incubated with secondary antibody and then with horseradish peroxidase-labeled streptavidin complex (DAKO). Distribution of peroxidase was revealed by incubating the sections in a solution containing $3 \%$ 3,3-diaminobenzidine tetrahydrochloride before being counterstained with hematoxylin and coverslipped. Negative control studies were performed using normal IgG to replace the primary antibody in appropriate concentration.

The histological analyses were performed in doubleblind manner. The immunoreactivity of five randomly selected epithelial or subepithelial areas $(400 x)$ on each section of the subjects were quantitatively scored using Image-Pro Plus 6.0 software and the average optical density (AOD) value was calculated.

\section{$q P C R$}

Periostin, TSLP, IL-4, IL-13, IFN $\gamma$ and IL-17a mRNA expression levels were evaluated by using quantitative realtime PCR (qRT-PCR) analysis, as previously described. Total RNA was extracted with TRizol reagent (Invitrogen) according to the manufacturer's instructions. The total RNA was then used to synthesize the first-strand cDNA using the 5X Prime Script Kit (Takara). Quantitative PCR was performed on the ABI7500 system (Invitrogen). For the relative quantification of $\mathrm{mRNA}$ expression, the expression of $\beta$-actin served as an internal control. Perfecta SYBR Green (Invitrogen) was used as a DNA intercalator dye to monitor amplified DNA quantification. mRNA expression level was calculated based on a standard curve.

The following primers were used: periostin forward: ACTTCCACGAGGTGTCCTAG; periostin reverse: CCTCCCATAATAGACTCAGAACA; TSLP forward: ACCGAGTTCAACAACACCGT; TSLP reverse: TCCGAATAGCCTGGGCAC; IL-4 forward: GCAGTTCTACAGCCACCATG; IL-4 reverse: ATGATCGTCTTTAGCCTTTCCA; IL-13 forward: AGCATGGTATGGAGCATCAAC; IL-13 reverse: CAGCATCCTCTGGGTCTTCT; TGF- $\beta 1$ forward: TGAACCGGCCTTTCCTGCTTCTCATG; TGF- $\beta 1$ reverse: GCGGAAGTCAATGTACAGCTGCCGC; IFN $\gamma$ forward: TGGAGACCATCAAGGAAGACAT; IFN $\gamma$ reverse: GCGACAGTTCAGCCATCAC; IL-17a forward: TCAACGCTGATGGGAACG; IL-17a reverse: TCTTGCTGGATGGGGACA.

\section{Western blot analysis}

Protein levels of periostin was evaluated by means of Western blot analysis as previously described. The protein extracts from nasal tissues underwent sodium dodecyl sulphate-polyacrylamide gel electrophoresis (SDSPAGE) and were transferred to a polyvinylidene fluoride membrane. The membranes were blocked and incubated with periostin primary antibody (Abcam, ab14041, 1:1,000), then incubated with the anti-Rabbit IgG HRP secondary antibody and detected using ECL detection reagent (Millipore). Western blot images were scanned and analysed using Gene'Tools from Syngene software (SYNGENE).

\section{ELISA}

Polyp tissues were homogenized with 2 times their weight of PBS ( $\mathrm{mL} / \mathrm{mg})$, and the final concentration was calculated from the weight of the tissue. The levels of periostin, TSLP, IL-4, IL-13 and TGF- $\beta 1$ in the homogenized supernatants of polyp tissues were measured by ELISA using a series of commercially available ELISA kits: periostin, R\&D Systems, DY3548B; TSLP, Abcam, ab192149; IL-4, R\&D Systems, DY205; IL-13, R\&D Systems, DY213; TGF- $\beta 1$, R\&D Systems, DB100B; according to the manufacturer's instructions.

\section{In vitro cell culture and stimulation}

Polyp tissues were collected from 4 randomly selected 
patients with CRSwNP, and HNECs were isolated, as we previously described (16). The NP tissue was cut into small pieces $(3-5 \mathrm{~mm}$ ), incubated with Dispase 2 (Sigma, \#D4693-1G) overnight and EDTA-Trypsin (0.5\%, GIBCO, Cat\# 25300-054) at $37{ }^{\circ} \mathrm{C}$ with $5 \% \mathrm{CO}_{2}, 15$ minutes, neutralized with DMEM (10\% FBS). The tissue pieces thoroughly dispersed into a single cell suspension, cultured in the 6 -well plate $\left(1 \times 10^{5}\right.$ cells), with DMEM $(10 \% \mathrm{FBS})$, at $37{ }^{\circ} \mathrm{C}$ with $5 \% \mathrm{CO}_{2}$, overnight. Drew the suspended cells, used for the non-HNECs. The adherent cells were HNECs. The HNECs were culture at air-liquid interface (ALI) using BEGMTM Bronchial Epithelial Cell Growth Medium (LONZA, USA) in the polyester membrane Transwell-clear inserts (Corning, Lowell, MA, USA), at $37{ }^{\circ} \mathrm{C}$ with $5 \% \mathrm{CO}_{2}$. After HNECs achieving 70-80\% confluence, $1 \mu \mathrm{g} / \mathrm{mL}$ periostin (R\&D Systems, 3548-F2050), $1 \mu \mathrm{g} / \mathrm{mL}$ TSLP (Abcam, ab201363), $100 \mathrm{ng} / \mathrm{mL}$ IL-4 (Peprotech, 200-0), 100 ng/mL IL-13 (Peprotech, 200-13) or $20 \mathrm{ng} / \mathrm{mL}$ TGF- $\beta 1$ (Peprotech, 100-21) were adding to bottom chamber medium alone or with $20 \mu \mathrm{M}$ PD98059 (the ERK pathway inhibitor, Sigma-Aldrich, P215), $100 \mu \mathrm{M}$ Leflunomide (the STAT6 pathway inhibitor, Sigma-Aldrich, L5025), $1 \mu \mathrm{g} / \mathrm{mL}$ the S-PN1 (the periostin inhibitor, Siriusbiotech, clone OC-20) and $20 \mu \mathrm{M}$ BAY 11-7082 (NF- $\kappa \mathrm{B}$ signaling inhibitor, Sigma-Aldrich, B5556) or $1 \mu \mathrm{g} / \mathrm{mL}$ Neutralizing Abs against TSLP (R\&D Systems, AF1398). In same experiments, the supernatant of treated HNECs was collected after 24 hours.

\section{Isolation of monocytes and culture of DCs}

Peripheral blood mononuclear cells (PBMCs) were isolated from buffy coats of blood from healthy donors by Ficoll density gradient centrifugation, as previously described (17). Monocytes were selected from PBMCs by anti-CD14 magnetic beads in a MACS column purification system (Miltenyi Biotech). To generate normal or activated DCs, the purified monocytes were cultured for 6-7 days in complete RPMI medium supplemented with $40 \mathrm{ng} / \mathrm{mL}$ GM-CSF and IL-4, alone or with the presence of HNECs supernatants as describing before. Half of the culture medium was replaced on day 3 and 5 .

\section{Mixed lymphocyte reaction}

Autologous blood CD45RA+CCR7+ naïve T cells $\left(2 \times 10^{5}\right.$ cells/well in 96-well plates; Pan T cell kit; Miltenyi Biotech) were co-culture by incubation with HNECs-activated DCs $\left(2 \times 10^{4}\right.$ cells/well $)$ in the presence of $10 \mathrm{U} / \mathrm{mL}$ IL- 2 and $10 \mathrm{ng} / \mathrm{mL}$ IL-7. Cells were harvested after 5 days, intracellular cytokines were respectively determined by FACS or quantitative RT-PCR analysis. FACS and quantitative RTPCR analysis was performed after re-stimulation of the cells with anti-CD3 Ab and anti-CD28 Ab for 24 hours.

\section{Flow cytometric staining and analysis}

For flow cytometry, living T cells were suspended in $400 \mu \mathrm{L}$ PBS with $1 \%$ bovine serum albumin for blocking unspecific staining. They were stained with the following anti-human monoclonal antibodies: anti-CD4-Pacific Blue, anti-IL4-APC, anti-IFN $\gamma$-PE-Cy5, and anti-IL-17A-PE-Cy7 (all antibodies and isotype controls were eBioscience) for intracellular staining. T cells were first stained for surface antigens. Following fixation and permeabilization with permeabilization/fixation buffer (BD Biosciences), these cells were stained with conjugated mAbs for cytokines according to the protocol of the permeabilization/ fixation kit. Samples were analyzed using a flow cytometer (Aira2, BD). Fluorescence was determined for all cells in each sample after debris, dead cells, and aggregates were excluded by forward angle and side scatter gating. Data compensation and analysis was performed using the accompanying software (FACSDiva, BD).

\section{Statistical analysis}

Data were expressed as the medians and analyzed using the nonparametric Mann Whitney $U$ test for unpaired comparisons and Spearman analysis except for additional note. In vitro assays, the data were expressed as the standard errors of the mean (SEM) of three independent experiments, one-way analysis of variance (ANOVA) and paired or unpaired Student's $t$-test were used for the statistical analysis. A P value less than 0.05 was considered as statistically significant.

\section{Results}

\section{Periostin immunoreactivity is associated with pathologic features and clinical parameters of CRSwNP}

This study includes 63 CRSwNP patients and 25 control subjects, the all demographic data are listed in Table 1. We firstly analyzed the histologic profile of polyp tissues in patients with periostin, HE and AB-PAS. Consequently, 
A

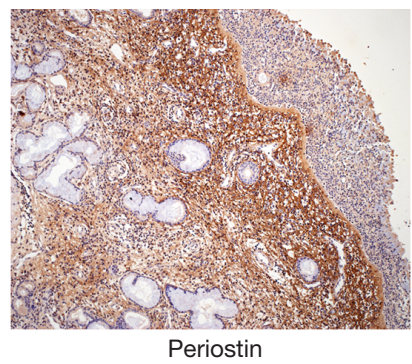

B

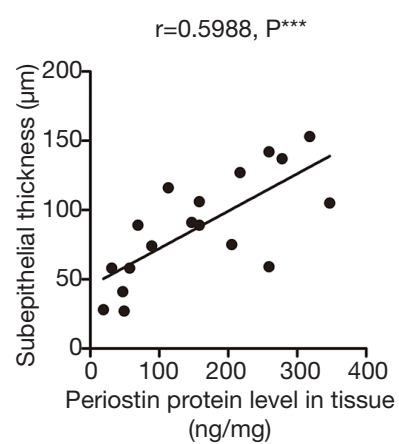

$E$

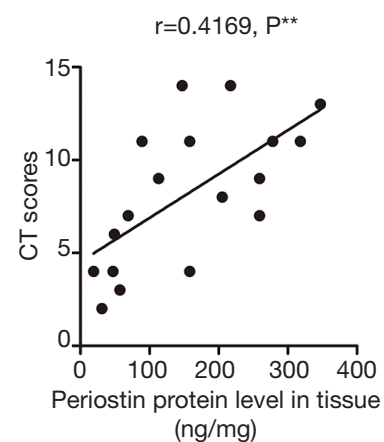

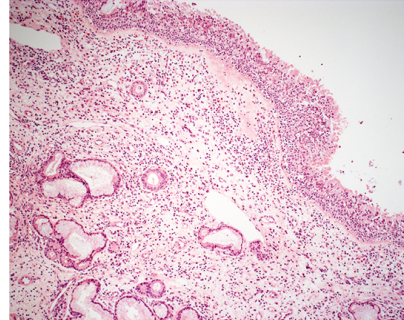

$\mathrm{HE}$

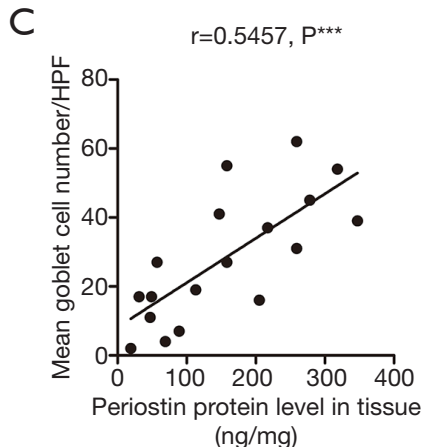

$\mathrm{F}$

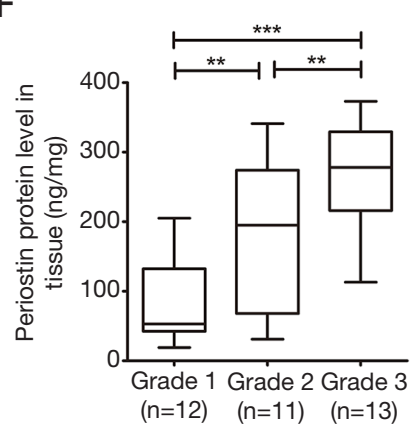

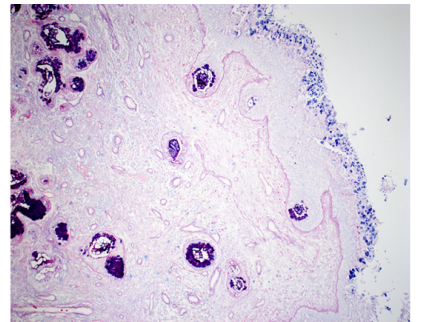

AB-PAS

$100 x$

D

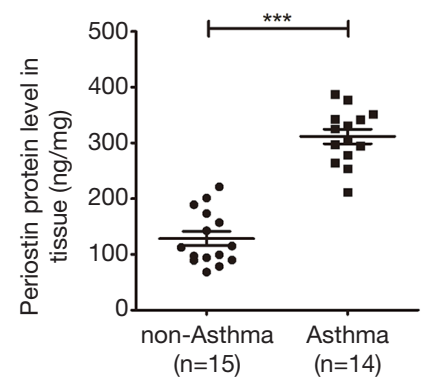

G

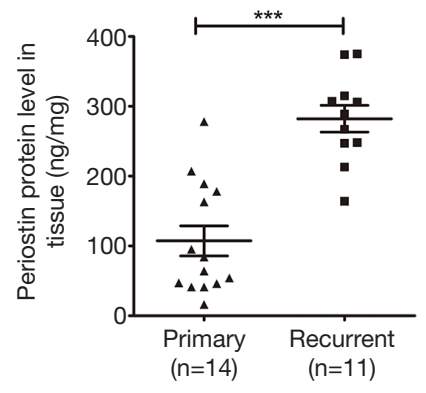

Figure 1 Periostin is associated with phenotype profiles and clinical severity of CRSwNP. (A) Representative graphs of NPs tissues staining with anti-periostin $\mathrm{Ab}, \mathrm{HE}$ and $\mathrm{AB}-\mathrm{PAS}$ (magnification, 100x); (B,C) the correlation of periostin concentrations and basement membrane thickness ( $r=0.5988)(B)$ and goblet cell hyperplasia $(r=0.5457)$ (C) in NPs tissues; (D,E,F,G) the periostin concentrations in NPs tissues was significantly associated with asthma comorbidity (D), CT score ( $\mathrm{r}=0.4169)(\mathrm{E})$. The periostin concentrations in different polyp sizes (F), and patients with initially diagnosed (primary) and recurrence (recurrence) nasal polyps $(\mathrm{G}) .{ }^{* *}, \mathrm{P}<0.01,{ }^{* * *}, \mathrm{P}<0.001$; as determined by Spearman correlation test or Mann Whitney U test. CRSwNP, chronic rhinosinusitis with nasal polyps; NP, nasal polyp; HE, hematoxylin and eosin; AB-PAS, Ally new blue and periodic acid-Schiff; CT, computed tomography; Ab, antibody.

we found that the protein immunoreactivity of periostin was associated with the basement membrane thickness (the indicator of subepithelial fibrosis), goblet cell hyperplasia (Figure $1 A, B, C)$. Next, we evaluated the relationship of periostin expressions and disease severity, and found that periostin levels were significantly associated with asthma comorbidity, CT scores, polyp size, and polyp recurrence after endoscopic surgery (Figure 1D,E,F,G).

\section{Elevated expression levels of periostin and TSLP in polyp tissues}

In the following study, our results showed that the levels of periostin and TSLP were significantly elevated in NPs compared with normal controls $(\mathrm{P}<0.001$, Figure $2 A, B, C, D)$. Also, the expressions of periostin and TSLP in immunoreactivity were also significantly increased in polyp 
A

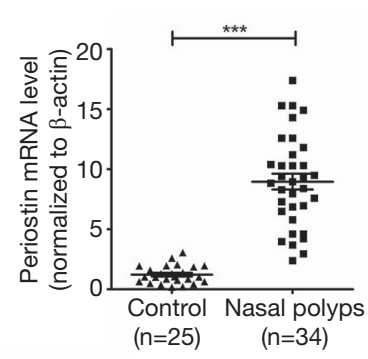

B

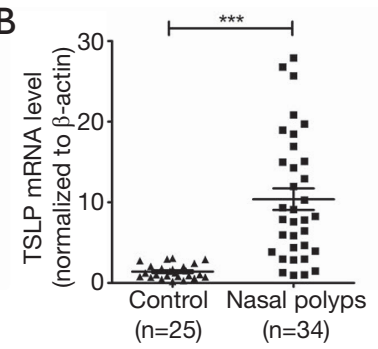

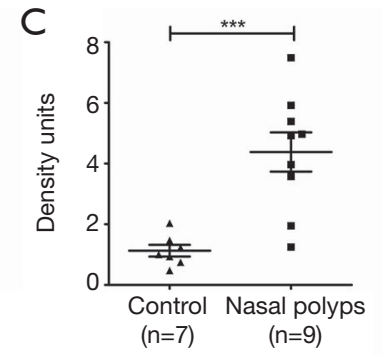

$E$

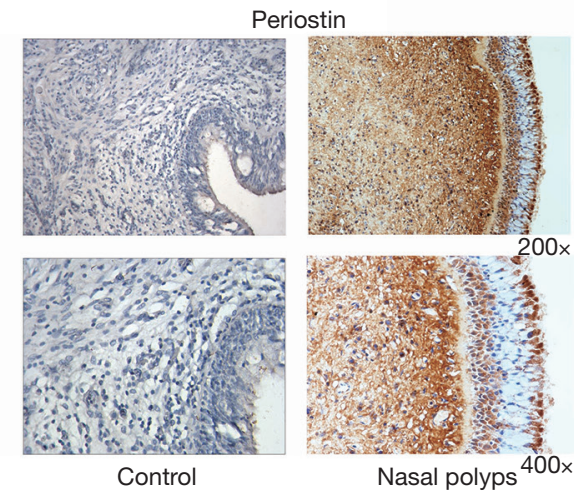

D

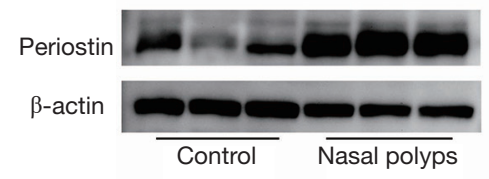

$\mathrm{F}$

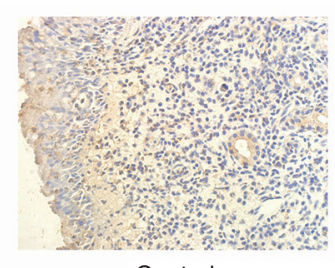

Control
TSLP

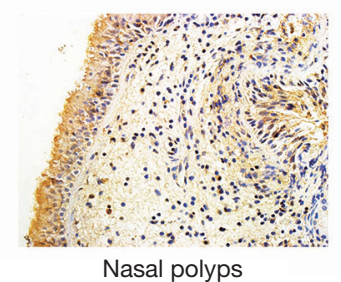

G

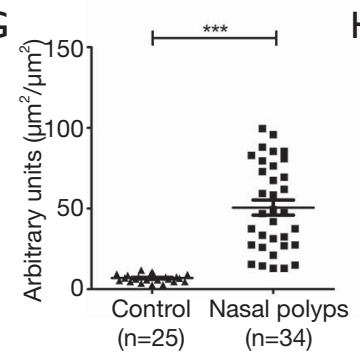

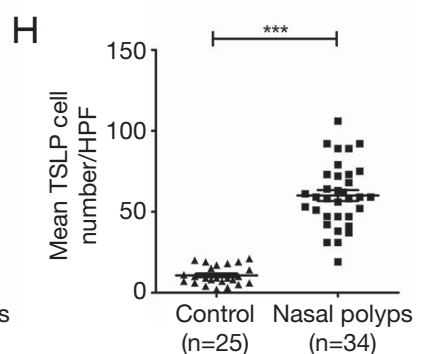

Figure 2 The expressions of periostin and TSLP in NPs were elevated than in the normal control. (A,B) The mRNA level of periostin (A) and TSLP (B) in the NPs tissues and the normal control; (C,D) the densitometric analysis (C), and the representative western blot results (D) of periostin protein in NPs tissues; (E,F) representative periostin (E) and TSLP (F) staining (200× and 400× magnification), and quantitative analysis of periostin $(\mathrm{G})$ and TSLP $(\mathrm{H})$ staining intensity in NPs tissues and control subjects. ${ }^{* * *}, \mathrm{P}<0.001$; as determined by Mann Whitney $\mathrm{U}$ test. TSLP, thymic stromal lymphopoietin; NP, nasal polyp.

tissues compared with control subjects $(\mathrm{P}<0.001$, Figure $2 E, F, G, H)$. Immunostaining showed that the expressions of periostin were wide distribution in polyp tissues, whereas the expressions of TSLP were mainly localized in the inflammatory cells and the epithelial regions. To clarity the source of periostin, we divided the single-cell suspension of NPs into HNECs and non-HNECs, then found the HNECs have higher mRNA and protein levels of periostin than non-HNECs $(\mathrm{P}<0.001$, Figure 3). Moreover, our study demonstrated that tissues periostin mRNA and protein concentrations levels were significantly correlated with TSLP expression in patients with CRSwNP ( $\mathrm{r}=0.5985$,
$\mathrm{P}<0.001$, Figure $4 A, \mathrm{r}=0.7241, \mathrm{P}<0.001$, Figure $4 B)$.

When we divided the CRSwNP into eosinophilic and noneosinophilic subsets according the tissue eosinophilia of polyp tissues. We observed significantly enhanced periostin and TSLP immunostaining in the eosinophilic compared with the noneosinophilic subset, even the two subsets had a similar tissue histopathology such as the goblet cell hyperplasia and collagen deposition (Figure 5A). The mRNA level and tissues protein concentrations of periostin and TSLP also were significantly higher in the eosinophilic polyp tissues $(\mathrm{P}<0.001$, Figure $5 B, C, D, E, F)$. However, the periostin $\mathrm{mRNA}$ and protein concentrations levels were also 


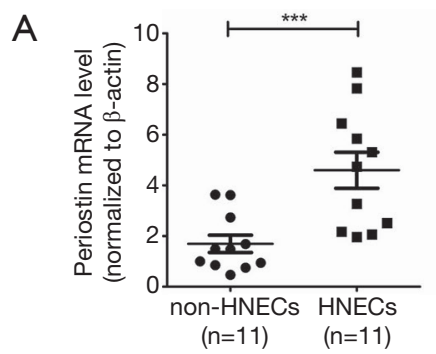

B

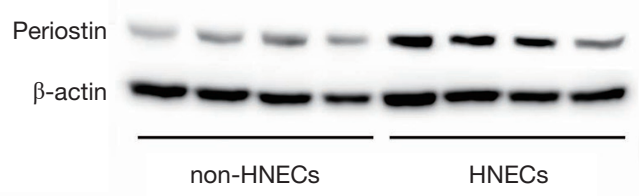

Figure 3 The expression of periostin is elevated in HNECs than non-HNECs. (A) The mRNA level of periostin in HNECs and nonHNECs; (B) the protein level of periostin in HNECs and non-HNECs. ***, $\mathrm{P}<0.001$; as determined by Mann Whitney U test. HNEC, human nasal epithelial cell.
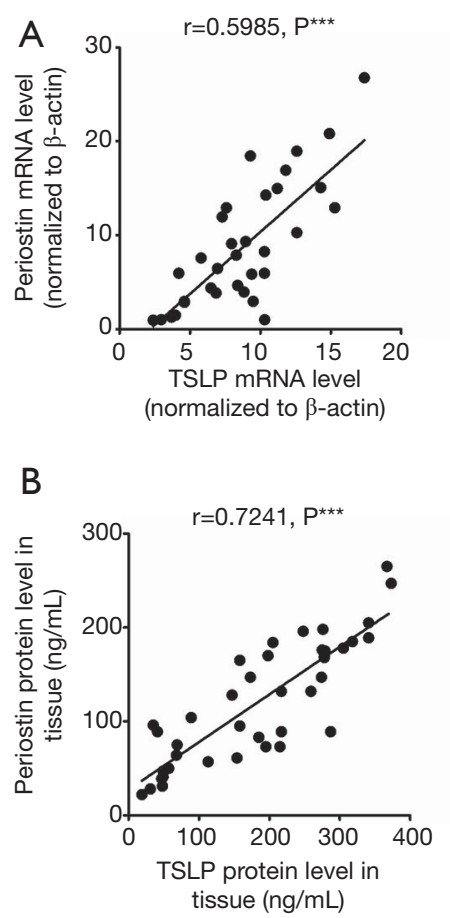
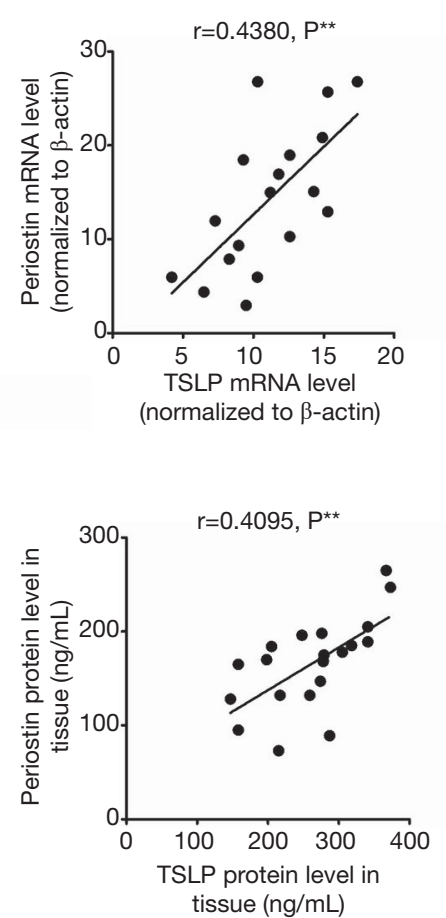
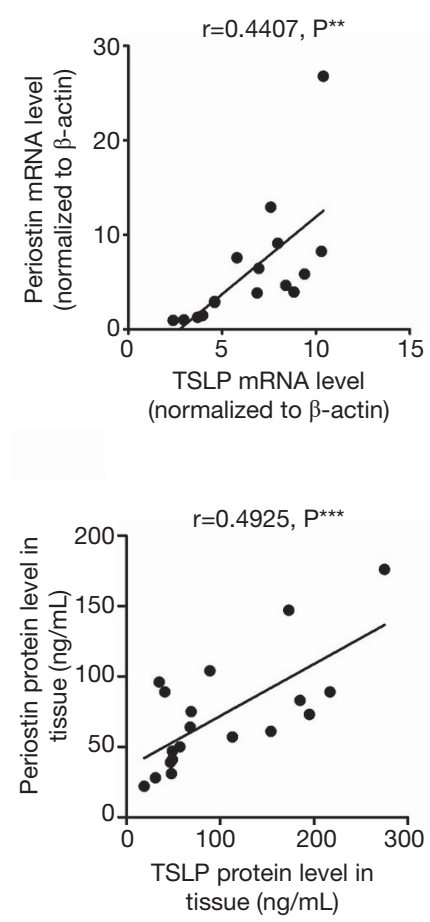

Figure 4 The expression of periostin in NPs were positively correlated with the TSLP. (A) The correlation of periostin and TSLP mRNA levels in total $(r=0.5985)$, eosinophilic $(r=0.4380)$ and noneosinophilic $(r=0.4407)$ subsets NPs tissues; (B) The correlation of periostin and TSLP protein levels in total $(\mathrm{r}=0.7241)$, eosinophilic $(\mathrm{r}=0.4095)$ and noneosinophilic $(\mathrm{r}=0.4925)$ subsets $\mathrm{NPs}$ tissues. ${ }^{* *}, \mathrm{P}<0.01,{ }^{* * *}, \mathrm{P}<0.001$; as determined by Spearman analysis. TSLP, thymic stromal lymphopoietin; NP, nasal polyp; NP, nasal polyp.

correlated with TSLP expression both in eosinophilic and noneosinophilic subsets polyp tissues $(\mathrm{r}=0.4380, \mathrm{P}<0.01$, $\mathrm{r}=0.4407, \mathrm{P}<0.01$, Figure $4 A ; \mathrm{r}=0.4095, \mathrm{P}<0.01, \mathrm{r}=0.4925$, $\mathrm{P}<0.001$, Figure $4 B$ ). These findings indicate that the periostin and TSLP is positive associated with eosinophilic inflammation in polyp tissues.

\section{Regulation of periostin and TSLP expression in cultured HNECs in vitro}

To clarify the mechanism of periostin and TSLP production in polyp tissues, we then examined the expression of periostin in response to several inflammatory cytokines existed in eosinophilic polyp tissues (Figure 6). As shown in Figure 6A, $B$, 

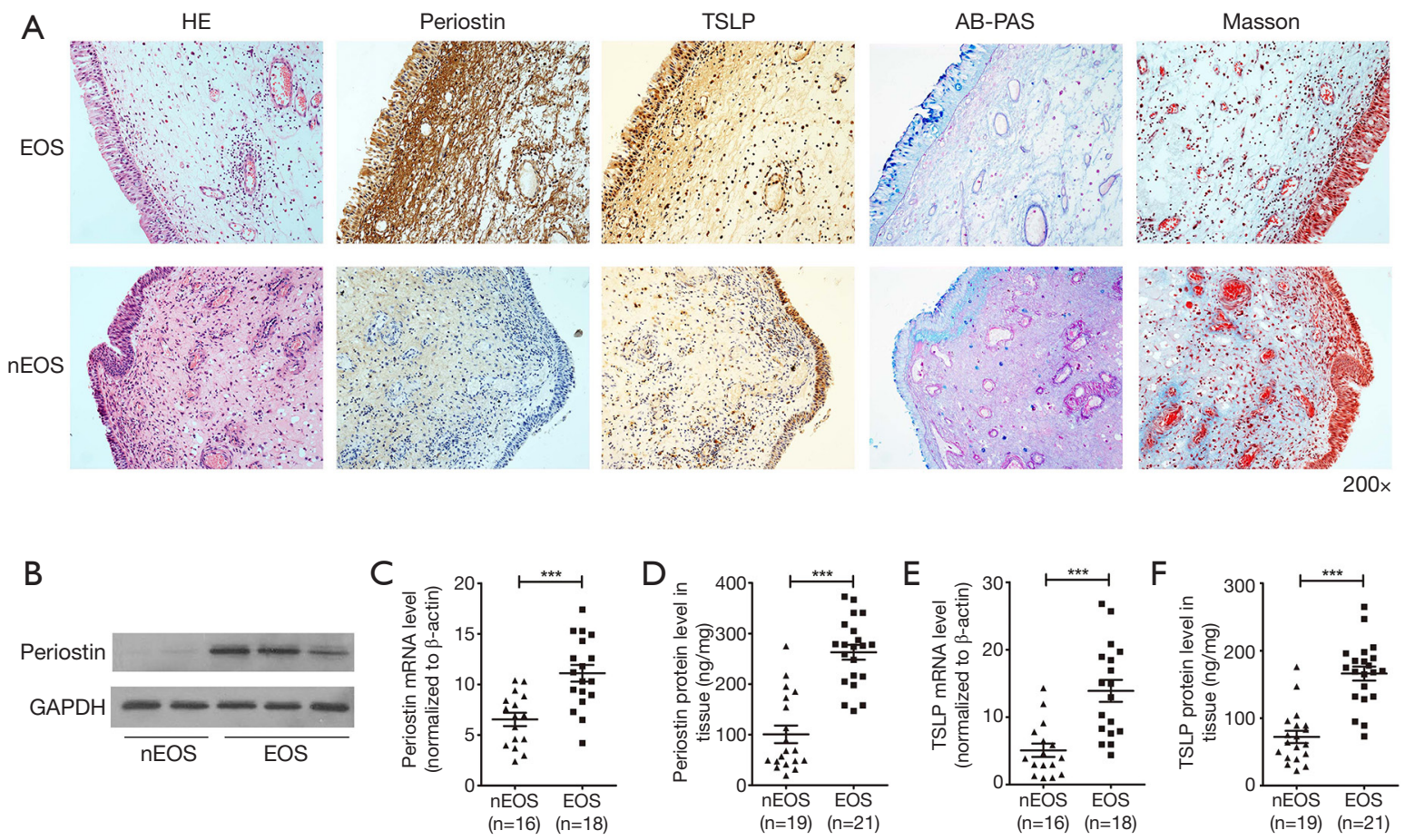

Figure 5 The periostin and TSLP levels were significantly higher in eosinophilic than noneosinophilic polyp tissues. (A) Representative HE, IHC staining with periostin and TSLP, AB-PAS and Masson staining (200× magnification); (B,C,D,E,F) the representative western blot results of periostin protein (B), the mRNA expression of periostin (C) and TSLP (E), ELISA analysis of periostin (D) and TSLP (F) in eosinophilic and noneosinophilic polyp tissues. ${ }^{* * *}, \mathrm{P}<0.001$ as determined by Mann Whitney $\mathrm{U}$ test. HE, hematoxylin and eosin; IHC, immunohistochemistry; AB-PAS, Alcian new blue and periodic acid-Schiff; TSLP, thymic stromal lymphopoietin; NP, nasal polyp; EOS, eosinophils.

periostin mRNA and protein levels were significantly increased after stimulating with IL-4, IL-13, TGF- $\beta 1$, in HNECs cultured in ALI. However, this upregulation was significantly inhibited by PD98059 (the ERK pathway inhibitor) and Leflunomide (the STAT6 pathway inhibitor) $(\mathrm{P}<0.05)$. In vivo experiments also demonstrated a positive correlation between periostin and IL-4, IL-13, TGF- $\beta 1$ expression in polyp tissues (Figure $6 E, F, G$ ). These findings suggest that inflammatory cytokines IL-4, IL-13, TGF- $\beta 1$ induced periostin production in HNECs and this was mediated by the extracellular-signal-regulated kinase (ERK) and the signal transducer and activator of transcription factor 6 (STAT6) pathways.

HNECs were stimulated with recombinant periostin alone or with the S-PN1 (the periostin inhibitor) in vitro, to observe the periostin's effect on TSLP. We found that recombinant periostin significantly increased the TSLP expression, whereas inhibited periostin could reduce TSLP production, and NF- $\mathrm{BB}$ signaling inhibitor BAY 11-7082 treatment also had an inhibitory effect $(\mathrm{P}<0.01$, Figure $6 C, D)$. These results indicated that inflammatory cytokines stimulated HNECs production periostin, and autocrine periostin directly induced TSLP production via NF-кB activation in HNECs.

\section{Nasal epithelial cell-derived periostin enhances $T h 2$ inflammation through TSLP-DCs axis}

Since several studies show that TSLP can activates or differentiates DCs to induce Th2-skewing (13), we tested whether HNECs from polyp tissues induced Th2 cells via the activation of DCs. Consequently, we found healthy control-derived DCs stimulated by periostin-treated HNECs supernatant, induced homologous CD4+ T cells to augment expression of IL-13 and IL-4, but not IFN- $\gamma$ and IL-17A in cell proportion and mRNA level, compared with untreated HNECs supernatant $(\mathrm{P}<0.05$, Figure 7$)$. Th2-skewing by periostin-treated HNECs supernatant 

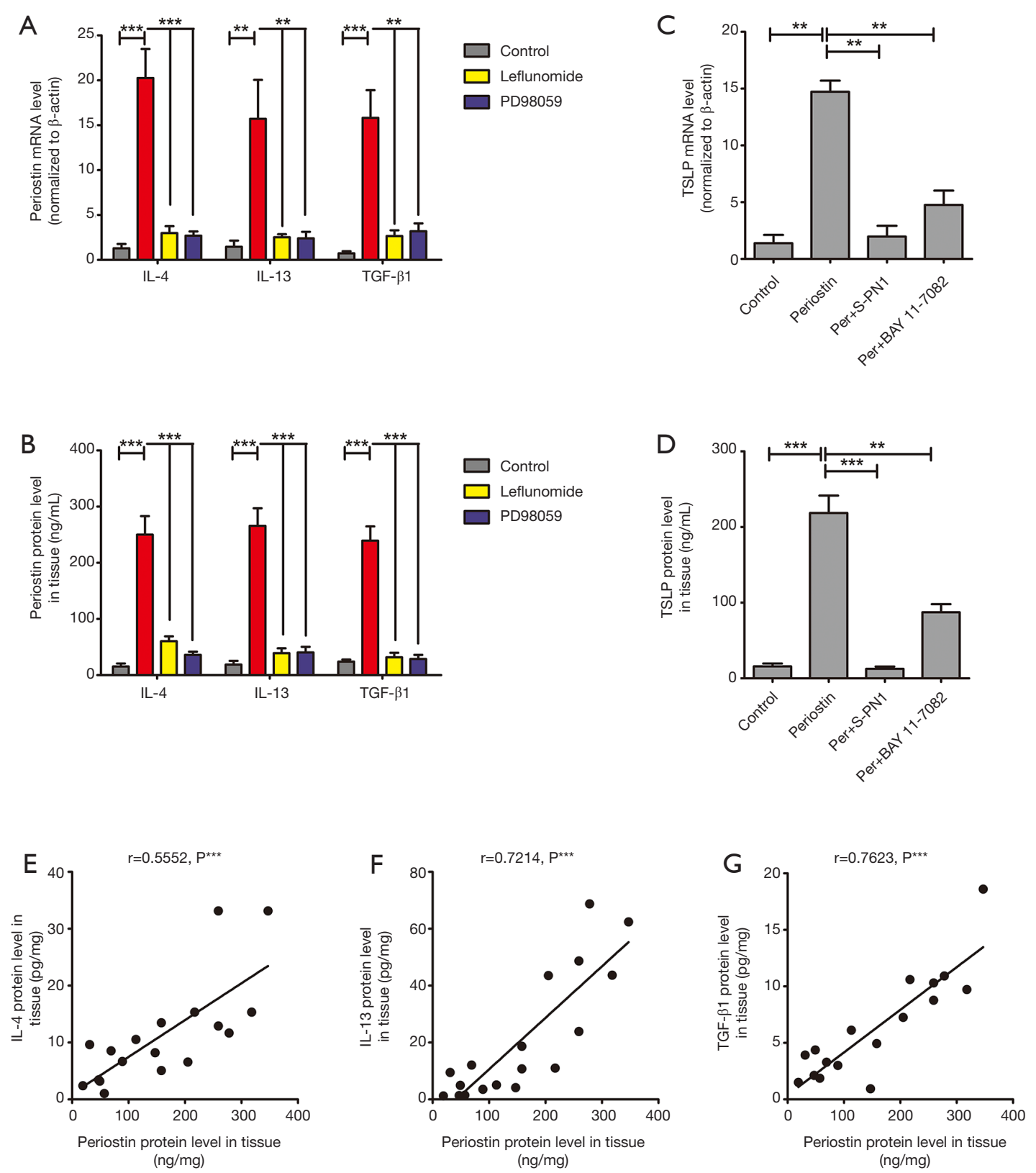

Figure 6 The regulation and underlying molecular mechanism of periostin and TSLP expression in vitro. Human nasal epithelial cells (HNECs) from NPs tissues, were cultured in ALI. (A,B) The mRNA level of periostin (A), and the ELISA analysis of periostin (B), which added of IL-4, IL-13, TGF- $\beta 1$ alone or with Leflunomide and PD98059 in the culture medium. (C,D) The mRNA level of TSLP (C), and the ELISA analysis of TSLP (D), which added of recombinant periostin alone or with S-PN1 or BAY 11-7082 in the culture medium. The periostin concentrations in NPs tissues was significantly associated with IL-4 (E), IL-13 (F), and TGF- $\beta 1$ (G) concentrations. **, P<0.01, ***, $\mathrm{P}<0.001$; as determined by Spearman analysis or Student's $t$-test and Spearman analysis Figures are representative of three different experiments. TSLP, thymic stromal lymphopoietin; NP, nasal polyp; ALI, air-liquid interface. 


\section{A}
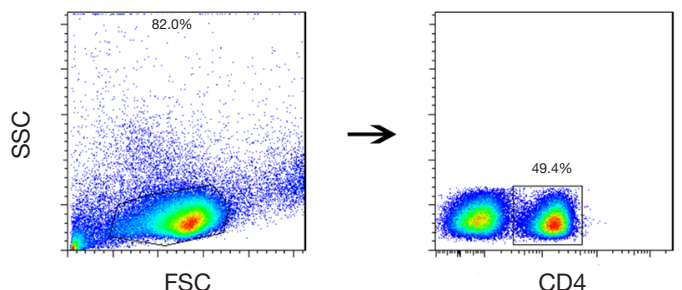

CD4
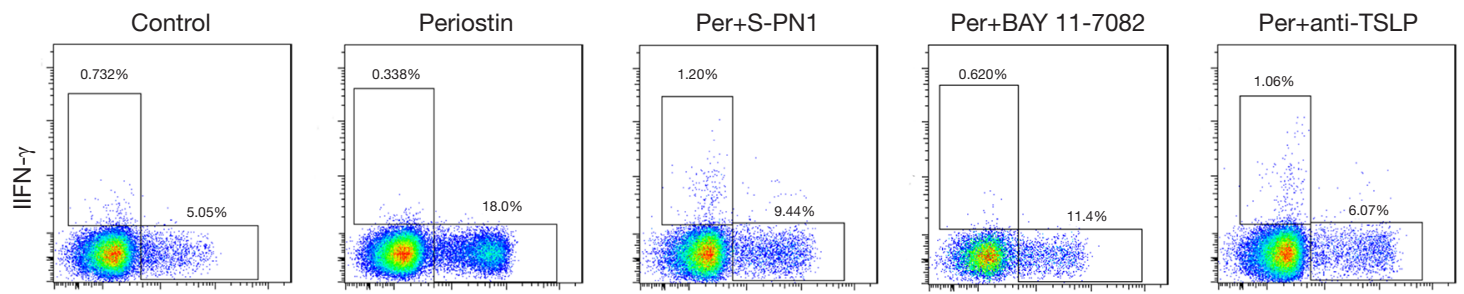

B
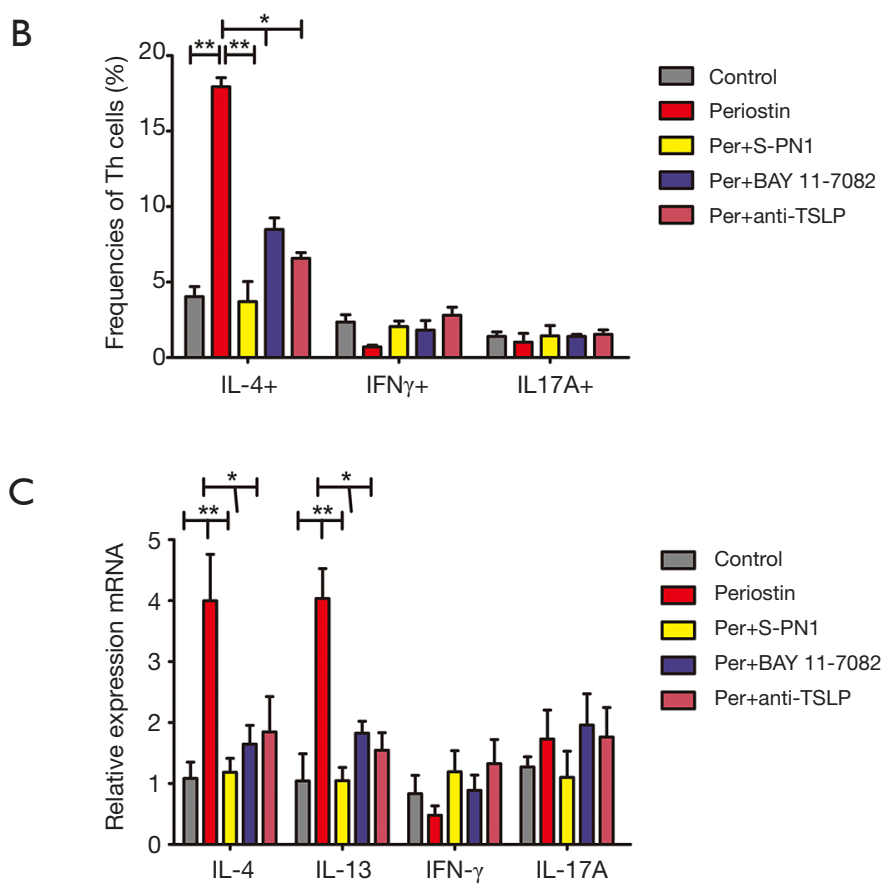

Figure 7 Periostin-treated HNECs supernatant enhances Th2 inflammation through TSLP activated DCs. HNECs were treated with recombinant periostin alone or with S-PN1, BAY 11-7082 or Ab anti-TSLP. DCs derived from CD14+ monocyte cells in PBMC of normal control, then cultured in complete RPMI medium alone or with the presence of HNECs supernatants as describing before. Autologous naïve $T$ cells were co-culture with HNECs-activated DCs 5 days. (A) Representative FACS graphs of intracellular cytokines IL-4, IFN $\gamma$ in the CD4+ T cells; (B) the frequencies analysis of IL-4, IFN $\gamma$, and IL-17A FACS; (C) the mRNA expression of IL-4, IL-13, IFN $\gamma$, and IL$17 \mathrm{~A}$ in the CD4+ $\mathrm{T}$ cells. ${ }^{*}, \mathrm{P}<0.05,{ }^{* *}, \mathrm{P}<0.01$; as determined by Student's $t$-test. Figures are representative of three different experiments. 
was partially inhibited by supplement of neutralizing Abs against TSLP, or the S-PN1 (the periostin inhibitor) and BAY 11-7082 (NF-kB signaling inhibitor) (Figure 7), indicating periostin-stimulated TSLP was involved in this event. These results demonstrated that the nasal epithelial cell-derived periostin enhances Th2 inflammation through TSLP-DCs axis.

\section{Discussion}

Periostin is a $90 \mathrm{KDa}$ secreted extracellular matrix (ECM) protein belonging to the fasciclin family $(18,19)$. Though interacting with other ECM proteins, periostin plays a wide variety of roles in tissue development and remodeling, and epithelial-mesenchymal transition in cancer and valvar heart disease $(9,20,21)$. In the previous study, periostin from fibroblast or bronchial epithelial cells, has been proposed to influence epidermal thickness and goblet cells mucus secretion in allergic inflammation $(12,22)$. Be consistent with the previous studies, we found that the expression of periostin was associated with the basement membrane thickness (the indicator of subepithelial fibrosis) and goblet cell hyperplasia in NPs tissues, suggesting that elevated periostin level may contribute to the characteristic tissues remodeling in NPs. Furthermore, our results also show that the increased expression of periostin is associated with a higher polyp size, asthma comorbidity, CT scores, and polyp recurrence after endoscopic surgery. In the polyp tissue, we found the expression of periostin was associated with eosinophilic infiltration (Figure 5), which was considered as the biomarker in predicting the recurrence of NPs (23). These findings indicate periostin may play a critical role in pathogenesis of CRSwNP patients.

Periostin is a recently characterized as a biomarker of Th2-skewing and eosinophilic inflammation disease such as CRSwNP, asthma, and atopic dermatitis (24-26). Previous studies show that fibroblasts and bronchial epithelial cells are the major secreting cells of periostin (11-13). In this study, we found that periostin was highly expressed in CRSwNP compared with normal nasal mucosa . However, Bachert et al. reported the in-situ hybridization showed periostin transcripts to be mainly expressed in the epithelium of NP tissue. We also found that HNECs had higher mRNA and protein levels of periostin than other cells in the NPs tissue $(\mathrm{P}<0.001$, Figure 3). Which suggests that epithelial cells are mainly sources of periostin deposited into the NPs tissue matrix accordance with another study $(27,28)$. Furthermore, we confirm that the concentration of periostin is connected with TSLP in NPs, which has been implicated in promoting Th2 responses in airway inflammation. TSLP is mainly localized in the inflammatory cells and the epithelial regions (29), combining with in vitro experiments showed that there is a periostin-TSLP-Th2 shewing signal pathway between in HNESs and immunes cells.

It is well known that CRSwNP is a complex disease containing distinct histopathologic endotypes (eosinophilic vs. noneosinophilic) (2-4). Eosinophilic CRSwNP subsets are characterized by dominant Th2 inflammation $(14,30,31)$. Here, we consistently found that periostin and TSLP immunoreactivity, as well as mRNA and protein levels, were significantly higher in eosinophilic CRSwNP than in noneosinophilic CRSwNP. The regulation of periostin has been widely examined and several mediators, including Th2 cytokines IL-4, IL-13; TGF- $\beta 1$, TGF- $\beta 3$; fibroblast growth factor; angiotensin II; vascular endothelial growth factor, have been reported to induce periostin production (32-34). However, there are few studies addressing the molecular mechanism in regulating periostin production in nasal epithelial cells. In this study, we demonstrated for the first time that the expression of IL-4, IL-13, and TGF- $\beta 1$ are positive associated with periostin concentration in polyp tissue. Furthermore, we demonstrated that IL-4, IL-13, and TGF- $\beta 1$ can stimulate HNECs secreting periostin thought ERK, STAT6 signaling pathway, and nasal epithelial cellderived periostin can promote the expression of TSLP by activating NF- $\mathrm{KB}$ signaling pathway. Several studies showed the activated epithelial cells can promote Th2 inflammation though secreted cytokines including TSLP, IL-25 and IL-33 (35). In this study, we found nasal epithelial cellderived periostin is able to stimulate TSLP production, and subsequently govern DCs to induce Th2 response by increasing expression of IL-4, IL-13 mRNA levels and IL-4 positive cells ratio. Our findings show autocrined periostin by nasal epithelial cells may promotes $\mathrm{Th} 2$ inflammation in CRSwNP through TSLP-DCs axis.

In conclusion, our findings show increased periostin expression in polyp tissue is associated with Th2 response, disease endotypes (eosinophilic vs. non-eosinophilic) and severity of CRSwNP patients, such as accompanying asthma. It suggests that periostin can be used as a predictor of eosinophilic NPs. We provided the novelty evidence that suggest that periostin can be induced by several proinflammatory cytokines in nasal epithelial cells thought ERK, STAT6 signaling pathway and autocrined periostin by nasal epithelial cells is subsequently able to promote 
Th2 inflammation via TSLP-DCs axis in vitro. Taken together, these findings may expand our understanding on the importance of periostin in the pathophysiology of CRSwNP, which may contribute to developing novel therapeutic strategy for the management of CRSwNP.

\section{Acknowledgements}

Funding: This study was supported by the National Natural Science Foundation of China (No. 81400451, 81470673, $81725004,81500781,81472999,81772699)$ and the Project Supported by Guangdong Natural Science Foundation (No. 2017A030313667, 8151012003000011) and Guangzhou Science and Technology Programme (No. 201605030003) and Shanghai Science and Technology Committee (No. 16410723600).

\section{Footnote}

Conflicts of Interest: The authors have no conflicts of interest to declare.

Ethical Statement: This study was approved by the Medical Ethics Committee of the First Affiliated Hospital, Sun Yatsen University \{No. [2014] C-054\}.

\section{References}

1. Fokkens WJ, Lund VJ, Mullol J, et al. EPOS 2012: European position paper on rhinosinusitis and nasal polyps 2012. A summary for otorhinolaryngologists. Rhinology 2012;50:1-12.

2. Hsu J, Peters AT. Pathophysiology of chronic rhinosinusitis with nasal polyp. Am J Rhinol Allergy 2011;25:285-90.

3. Akdis CA, Bachert C, Cingi C, et al. Endotypes and phenotypes of chronic rhinosinusitis: a PRACTALL document of the European Academy of Allergy and Clinical Immunology and the American Academy of Allergy, Asthma \& Immunology. J Allergy Clin Immunol 2013;131:1479-90.

4. Bachert C, Akdis CA. Phenotypes and Emerging Endotypes of Chronic Rhinosinusitis. J Allergy Clin Immunol Pract 2016;4:621-8.

5. Bao Y, Chen J. Chinese Guideline on allergen immunotherapy for allergic rhinitis. J Thorac Dis 2017;9:4607-50.

6. Liao B, Cao PP, Zeng M, et al. Interaction of thymic stromal lymphopoietin, IL-33, and their receptors in epithelial cells in eosinophilic chronic rhinosinusitis with nasal polyps. Allergy 2015;70:1169-80.

7. Hong HY, Chen FH, Sun YQ, et al. Local IL-25 contributes to Th2-biased inflammatory profiles in nasal polyps. Allergy 2018;73:459-69.

8. Conway SJ, Izuhara K, Kudo Y, et al. The role of periostin in tissue remodeling across health and disease. Cell Mol Life Sci 2014;71:1279-88.

9. Bentley JK, Chen Q, Hong JY, et al. Periostin is required for maximal airways inflammation and hyperresponsiveness in mice. J Allergy Clin Immunol 2014;134:1433-42.

10. Takayama G, Arima K, Kanaji T, et al. Periostin: a novel component of subepithelial fibrosis of bronchial asthma downstream of IL-4 and IL-13 signals. J Allergy Clin Immunol 2006;118:98-104.

11. Suzaki I, Kawano S, Komiya K, et al. Inhibition of IL13 -induced periostin in airway epithelium attenuates cellular protein expression of MUC5AC. Respirology 2017;22:93-100.

12. Masuoka M, Shiraishi H, Ohta S, et al. Periostin promotes chronic allergic inflammation in response to Th2 cytokines. J Clin Invest 2012;122:2590-600.

13. Taniguchi K, Arima K, Masuoka M, et al. Periostin controls keratinocyte proliferation and differentiation by interacting with the paracrine IL-1alpha/IL-6 loop. J Invest Dermatol 2014;134:1295-304.

14. Wen W, Liu W, Zhang L, et al. Increased neutrophilia in nasal polyps reduces the response to oral corticosteroid therapy. J Allergy Clin Immunol 2012;129:1522-8.e5.

15. Xiao L, Wei Y, Zhang YN, et al. Increased IL-21 expression in chronic rhinosinusitis with nasalpolyps. Clin Exp Allergy 2015;45:404-13.

16. Lai Y, Chen B, Shi J, et al. Inflammation-mediated upregulation of centrosomal protein 110, a negative modulator of ciliogenesis, in patients with chronic rhinosinusitis. J Allergy Clin Immunol 2011;128:12071215.e1.

17. Zhao Q, Xiao X, Wu Y, et al. Interleukin-17-educated monocytes suppress cytotoxic T-cell function through B7$\mathrm{H} 1$ in hepatocellular carcinoma patients. Eur J Immunol 2011;41:2314-22.

18. Coutu DL, Wu JH, Monette A, et al. Periostin, a member of a novel family of vitamin K-dependent proteins, is expressed by mesenchymal stromal cells. J Biol Chem 2008;283:17991-8001.

19. Lorts A, Schwanekamp JA, Baudino TA, et al. Deletion of periostin reduces muscular dystrophy and fibrosis in mice by modulating the transforming growth factor-beta 
pathway. Proc Natl Acad Sci U S A 2012;109:10978-83.

20. Chen G, Nakamura I, Dhanasekaran R, et al. Transcriptional Induction of Periostin by a Sulfatase 2-TGFbeta1-SMAD Signaling Axis Mediates Tumor Angiogenesis in Hepatocellular Carcinoma. Cancer Res 2017;77:632-45.

21. Kanisicak O, Khalil H, Ivey MJ, et al. Genetic lineage tracing defines myofibroblast origin and function in the injured heart. Nat Commun 2016;7:12260.

22. Shiono O, Sakuma Y, Komatsu M, et al. Differential expression of periostin in the nasal polyp may represent distinct histological features of chronic rhinosinusitis. Auris Nasus Larynx 2015;42:123-27.

23. Stevens WW, Schleimer RP, Kern RC. Chronic Rhinosinusitis with Nasal Polyps. J Allergy Clin Immunol Pract 2016;4:565-72.

24. Li W, Gao P, Zhi Y, et al. Periostin: its role in asthma and its potential as a diagnostic or therapeutic target. Respir Res 2015;16:57.

25. Murota H, Lingli Y, Katayama I. Periostin in the pathogenesis of skin diseases. Cell Mol Life Sci 2017;74:4321-28.

26. Jia G, Erickson RW, Choy DF, et al. Periostin is a systemic biomarker of eosinophilic airway inflammation in asthmatic patients. J Allergy Clin Immunol 2012;130:647654.e10.

27. Wang M, Wang XD, Zhang N, et al. Association of periostin expression with eosinophilic inflammation in nasal polyps. J Allergy Clin Immunol 2015;136:17001703.e9.

28. Kim DW, Kulka M, Jo A, et al. Cross-talk between human mast cells and epithelial cells by IgE-mediated periostin production in eosinophilic nasal polyps. J Allergy Clin Immunol 2017;139:1692-1695.e6.

29. Lu X, Fu H, Han F, et al. Lipoxin A4 regulates PM2.5induced severe allergic asthma in mice via the Th1/Th2 balance of group 2 innate lymphoid cells. J Thorac Dis 2018;10:1449-59.

30. Wei Y, Xia W, Ye X, et al. The antimicrobial protein short palate, lung, and nasal epithelium clone 1 (SPLUNC1) is differentially modulated in eosinophilic and noneosinophilic chronic rhinosinusitis with nasal polyps. J Allergy Clin Immunol 2014;133:420-8.

31. Robinson D, Humbert M, Buhl R, et al. Revisiting Type 2-high and Type 2-low airway inflammation in asthma: current knowledge and therapeutic implications. Clin Exp Allergy 2017;47:161-75.

32. Gordon ED, Sidhu SS, Wang ZE, et al. A protective role for periostin and TGF-beta in IgE-mediated allergy and airway hyperresponsiveness. Clin Exp Allergy 2012;42:144-55.

33. Norris RA, Moreno-Rodriguez R, Markwald RR, et al. The many facets of the matricelluar protein periostin during cardiac development, remodeling, and pathophysiology. J Cell Commun Signal 2009;3:275-86.

34. Noguchi T, Nakagome K, Kobayashi T, et al. Periostin upregulates the effector functions of eosinophils. J Allergy Clin Immunol 2016;138:1449-1452.e5.

35. Gon Y, Hashimoto S. Role of airway epithelial barrier dysfunction in pathogenesis of asthma. Allergol Int 2018;67:12-7.
Cite this article as: Wei Y, Ma R, Zhang J, Wu X, Yu G, Hu X, Li J, Liu Z, Ji W, Li H, Wen W. Excessive periostin expression and Th2 response in patients with nasal polyps: association with asthma. J Thorac Dis 2018;10(12):6585-6597. doi: 10.21037/ jtd.2018.11.12 